\title{
Identification of prognostic biomarkers for malignant melanoma using microarray datasets
}

\author{
GUANYU LIN, GUOQIAN YIN, YUYONG YAN and BOJIE LIN \\ Department of Plastic and Aesthetic Surgery, The First Affiliated Hospital of Guangxi Medical University, \\ Nanning, Guangxi 530021, P.R. China
}

Received May 6, 2019; Accepted September 6, 2019

DOI: $10.3892 / \mathrm{ol} .2019 .10914$

\begin{abstract}
Malignant melanoma is one of the most common types of cancer worldwide. Efforts have been made to elucidate the pathology of malignant melanoma. However, its molecular mechanisms remain unclear. Therefore, the microarray datasets GSE3189, GSE4570 and GSE4587 from the Gene Expression Omnibus database were used for the elucidation of candidate genes involved in the initiation and progression of melanoma. Assessment of the microarray datasets led to the identification of differentially expressed genes (DEGs), which were subsequently used for function enrichment analysis. These data were utilized in the construction of the protein-protein interaction network and module analysis was conducted using STRING and Cytoscape software. The results of these analyses led to the identification of a total of 182 DEGs, including 52 downregulated and 130 upregulated genes. The functions and pathways found to be enriched in the DEGs were GTPase activity, transcription from RNA polymerase II promoter, apoptotic processes, cell adhesion, membrane related pathways, calcium signaling cascade and the PI3K-Akt signaling pathway. The identified genes were demonstrated to belong to a set of 10 hub genes biologically involved in proliferation, apoptosis, cytokinesis, adhesion and migration. Survival analysis and Oncomine database analysis revealed that the calmodulin gene family, $B A X$ and $V E G F A$ genes, may be associated with the initiation, invasion or recurrence of melanoma. In conclusion, the DEGs and hub genes identified in the present study may be used to understand the molecular pathways involved in the initiation and progression of malignant melanoma. Furthermore, the present study may aid in the identification of possible targets for the diagnosis and treatment of melanoma.
\end{abstract}

Correspondence to: Dr Bojie Lin, Department of Plastic and Aesthetic Surgery, The First Affiliated Hospital of Guangxi Medical University, 6 Shuangyong Road, Nanning, Guangxi 530021, P.R. China E-mail: linbojie@aliyun.com

Key words: malignant melanoma, prognosis, differentially expressed genes, microarray, $B A X$

\section{Introduction}

Melanoma is a malignant skin tumor derived from melanocytes, which has a high degree of malignancy and leads to high mortality. During the past 40 years, its incidence, as well as the proportion of mid- to late-stage tumors and infeasibility of surgery have increased worldwide (1).

Previous studies have demonstrated that abnormal expression and mutations of genes and proteins, including cell division cycle associated 8 (encoded by $C D C A 8$ ), telomerase reverse transcriptase $(T E R T)$, B-Raf proto-oncogene $(B R A F)$ and various tumor suppressor genes are involved in the initiation and progression of melanoma. For example, it has been reported that the $C D C A 8$ gene is capable of promoting the malignant progression of cutaneous melanoma and is associated with poor prognosis (2). TERT promoter mutations have also been identified in up to $50 \%$ of cutaneous melanoma cases in the global population; however, their incidence in Asian populations remains unclear (3). $B R A F$ mutations, particularly those located at codon 600 , have been observed in $50 \%$ of malignant melanoma cases worldwide (4). Furthermore, overexpression of $B R A F$ and hypermethylation of Ras binding proteins have been revealed to be associated with poor prognosis in patients with malignant melanoma $(5,6)$. Melanoma mortality remains high due to the absence of efficient diagnostic techniques at the initial stages of the disease. Therefore, understanding the mechanisms involved in the initiation, proliferation and recurrence of this type of cancer at the molecular level is essential for the development of more effective diagnostic and treatment strategies.

Over the past few decades, microarray analyses as well as bioinformatics studies have been increasingly favored for the screening of genetic changes at the genomic level. These identification methods may be employed for the determination of differentially expressed genes (DEGs), as well as functions that may be involved in melanoma initiation and progression (7). However, the reliability of independent microarray analyses may not be high owing to the rate of false positives. Therefore, in the present study, three different mRNA microarray datasets were obtained from the Gene Expression Omnibus (GEO) database. These datasets were analyzed for the determination of DEGs between malignant melanoma and normal nevi tissues. Thereafter, Gene Ontology (GO) and Kyoto Gene and Genomic Encyclopedia (KEGG) analyses, 
as well as protein-protein interaction (PPI) network analysis were conducted to identify molecular processes associated with melanoma development and progression. Altogether, 182 DEGs and 10 hub genes were indicated as potential biomarkers of melanoma.

\section{Materials and methods}

Data from the microarray analyses. GEO (http://www.ncbi. nlm.nih.gov/geo) (8) is a publicly available function-related genomics repository containing high-throughput gene expression data, ChIP-seq data, as well as microarrays. Three gene datasets, GSE3189 (9), GSE4570 (10) and GSE4587 (11), were downloaded from the GEO database. GSE3189 and GSE4570 were based on the ArrayGPL96 platform (Affymetrix Human Genome U133A Array), whereas GSE4587 was based on the GPL570 platform (Affymetrix Human Genome U133 Plus 2.0). The probes were later converted to their analogous gene symbols using platform information. The GSE3189 dataset comprised 45 melanoma tissue samples and 18 normal nevi tissue samples, GSE4570 contained 6 melanoma samples and 2 nevi samples, and GSE4587 contained 7 melanoma samples and 8 nevi samples.

Identification of DEGs. GEO2R (http://www.ncbi.nlm.nih. gov/geo/geo2r) was used for the screening of DEGs between melanoma and normal nevi tissue samples. GEO2R is a web tool used in interaction studies for the comparison of various datasets in a GEO series to identify DEGs. As mentioned above, the most common limitation of microarray analyses is the presence of false positives, which may be limited by adjusting the $\mathrm{P}$-values and calculating the Benjamini-Hochberg false discovery rate (12). These adjustments led to the identification of statistically significant genes. Probe sets that had either no corresponding gene symbols or genes with numerous probe sets were eliminated or averaged, respectively. DEGs of logarithmic fold change value $>1$ were selected in the present study. $\mathrm{P}<0.05$ was considered to indicate a statistically significant difference.

KEGG and GO enrichment analysis of the DEGs. The Database for Annotation, Visualization and Integrated Discovery (DAVID; version 6.8; http://david.ncifcrf.gov) (13) is an online bioinformatics database that integrates biological data with analytic tools, and offers substantial gene- and protein-related information, thus contributing to the extraction of biological data. KEGG database is a online tool used to study advanced functions and biological processes of genes via high-throughput sequencing (14). GO, a significant web-based tool used for annotating genes and analyzing the biological processes that these genes are involved in, was also employed for DEG enrichment (15). The biological functions of the DEGs were analyzed using DAVID. $\mathrm{P}<0.05$ was considered to indicate a statistically significant difference.

Construction of a PPI network and module analysis. The Search Tool for the Retrieval of Interacting Genes (STRING; version 10.5; http://string-db.org) database was used to analyze the PPI network of genes (16). Analysis of functionally relevant interactions among the proteins encoded by
DEGs may provide valuable insights into the mechanisms of genesis or progression of various diseases. In the present study, the STRING database was used for the construction of the DEG PPI network. A statistically relevant interaction was defined using STRING (combined score $>0.4$ ). Cytoscape (version 3.7.0), an open access bioinformatics software, is a platform used to study networks of molecular interactions (17). Furthermore, Molecular Complex Detection (MCODE; version 1.5.1), a Cytoscape plugin, clusters a given network based on topology for the determination of compact connected portions (18). Cytoscape was used for the identification of the PPI network, while MCODE was used to identify the most significant interaction. The MCODE selection criteria were as follows: i) MCODE score $>5$; ii) MCODE degree cut-off level $=2$; iii) node score cut-off level $=0.2$; iv) $\max$ depth $=100$ and v) $\mathrm{k}$-score $=2$.

Hub genes selection and analysis. After the construction of the PPI network, genes (MCODE degrees $\geq 10$ using Cytoscape) were identified as hub genes. The gene network and genes that were co-expressed within this network were determined using cBioPortal (http://www.cbioportal.org) $(19,20)$. Investigation of the biological processes associated with the hub genes was conducted using the Biological Networks Gene Oncology (version 3.0.3) Cytoscape plugin (21). The overall survival and disease-free survival analyses of the hub genes were conducted using the Kaplan-Meier function in cBioPortal. The gene expression levels of the hub genes between melanoma and normal nevi tissues were evaluated using Oncomine, an online database (http://www.oncomine.com) $(22,23)$. Differences in expression between melanoma and normal nevi tissues were analyzed by t-test. $\mathrm{P}<0.05$ was considered to indicate a statistically significant difference.

\section{Results}

$D E G$ identification in melanoma. With logarithmic fold change value $>1$ and $\mathrm{P}<0.05$, the DEGs $(2,484$ in GSE3189, 1,300 in GSE4570 and 6,759 in GSE4587) were identified. The intersection of the 3 datasets comprised of 182 genes, 52 of which were downregulated and 130 were upregulated between melanoma and normal nevi tissues, as demonstrated in the Venn diagram (Fig. 1A).

Enrichment analyses of the DEGs using KEGG and GO. Biological classification of the DEGs, achieved via functional and pathway enrichment analyses, was performed using DAVID. The results of the GO analysis revealed that the DEGs in biological processes were enriched in transcription from RNA polymerase II promoter, cell adhesion, GTPase activity, apoptotic processes and transcription. At the molecular level, changes in functions were mainly enriched in actin and protein kinase binding. Regarding the 'cellular component', DEGs were mainly enriched in the cell membrane, cytoskeleton and extracellular region (Table I). KEGG pathway analysis demonstrated that the downregulated DEGs were mostly enhanced in the estrogen signaling cascade, melanogenesis and the calcium signaling pathway, whereas the upregulated DEGs were mostly enriched in microRNAs in focal adhesion and pathways in cancer, as well as the PI3k-Akt signaling pathway. 


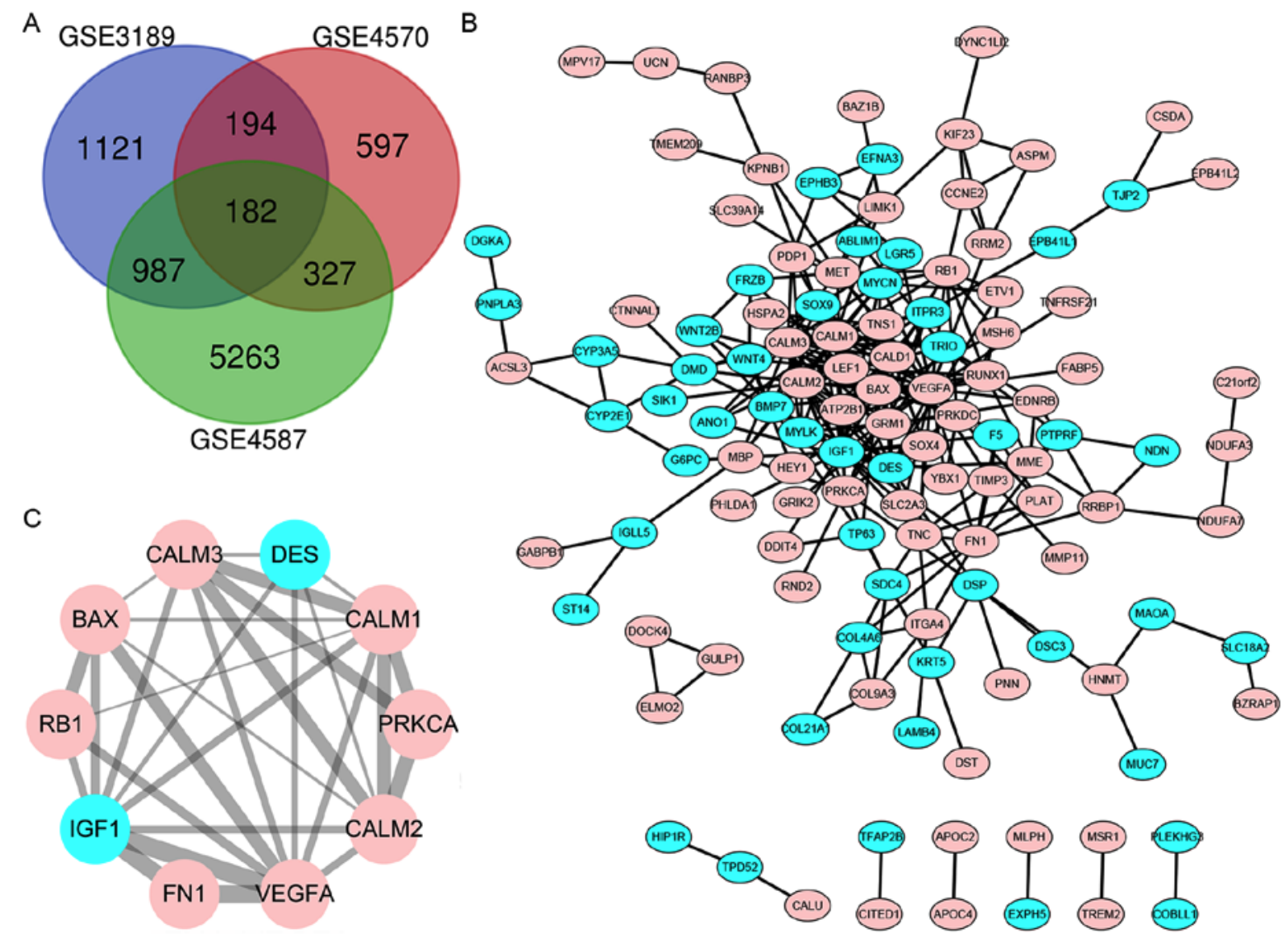

Figure 1. Venn diagram of the three gene microarray datasets, PPI network obtained using bioinformatics algorithms and statistically significant DEG module. (A) DEGs with a fold change $>2$ and P $<0.05$ were selected among the gene expression profiling GSE4587, GSE3189 and GSE4570 datasets. A common set of 182 genes was identified at the intersection of the 3 datasets. (B) Cytoscape was used for the construction of the PPI network of the DEGs. (C) Module of maximum relevance, consisting of 10 nodes and 29 edges, extracted from PPI network. Genes with upregulated expression are shown in pink, whereas genes with downregulated expression are shown in blue. PPI, protein-protein interaction; DEG, differentially expressed gene; DES, desmin; VEGFA, vascular endothelial growth factor A, CALM1, calmodulin 1; CALM2, calmodulin 2; CALM3, calmodulin 3; FN1, fibronectin 1; PRKCA, protein kinase C $\alpha$; IGF1, insulin-like growth factor 1; RB1, retinoblastoma transcriptional corepressor 1 .

Construction of the PPI network and module analysis. The DEG PPI network was constructed to find novel protein interactions. A total of 122 nodes and 266 protein interaction pairs were identified (Fig. 1B). The most significant interaction of 10 nodes and 29 protein interaction pairs was identified using Cytoscape (Fig. 1C).

Selection and analysis of hub genes. Ten genes (MCODE degrees $\geq 10$ using Cytoscape) were revealed as hub genes. The names, acronyms and roles of these hub genes are presented in Table II. The cBioPortal online platform was used to analyze the hub gene network, as well as their co-expression genes (Fig. 2). The cBioPortal network contains 60 nodes, including 10 hub genes and the 50 most frequently altered neighbor genes. The results of the hub gene biological process analysis are demonstrated in Fig. 3. The biological processes 'cell division', 'cytokinesis, release of sequestered ion into cytosol', 'release of sequestered calcium ion into cytosol by sarcoplasmic reticulum', 'ryanodine-sensitive calcium-release channel activity' and 'ion transmembrane transporter activity' were significantly enriched. Subsequently, survival analysis of the hub genes was performed using Kaplan-Meier analysis, as presented in Fig. 4. Melanoma patients with $B A X$ alterations were identified to have poor disease-free as well as overall survival (Fig. 4), indicating that $B A X$ may serve a significant role in the initiation or progression of melanoma. Oncomine analysis of melanoma and normal nevi tissues revealed that $B A X$ was significantly overexpressed in melanoma tissues in the different datasets (Fig. 5). In the Oncomine database, the mRNA expression levels of $B A X(\mathrm{P}<0.0001), C A L M 1$ [which encodes calmodulin (CaM1)] $(\mathrm{P}<0.0001), C A L M 3$ (CaM3; $\mathrm{P}<0.0001$ ), FN1 (fibronectin 1; $\mathrm{P}<0.0001), P R K C A$ (protein kinase $\mathrm{C} \alpha ; \mathrm{P}=0.0320$ ), RBl (RB transcriptional corepressor 1; $(\mathrm{P}<0.0001)$ and VEGFA (vascular endothelial growth factor $\mathrm{A}$; $\mathrm{P}<0.0001)$ genes were demonstrated to be higher in melanoma tissues than in the normal nevi tissues. By contrast, the mRNA expression level of IGF1 (insulin-like growth factor 1) was found to be lower in melanoma tissues. There was no statistically difference in the expression of $D E S(\mathrm{P}=0.0628)$ and CALM2 $(\mathrm{P}=0.0552)$ between melanoma and normal nevi tissues.

\section{Discussion}

Melanoma is a highly metastatic type of cancer, which exhibits strong resistance to both chemotherapy and radiotherapy (24-26). It has been observed to develop rapidly during the early phase. Melanoma cells acquire mutations during this 
Table I. Enrichment study of DEGs between malignant melanoma tissue and normal nevus tissue.

A. Downregulated DEGs

Term and description

Count in gene set $\mathrm{P}$-value

$3.39 \times 10^{-5}$

$4.72 \times 10^{-5}$

GO:0030801 Positive regulation of cyclic nucleotide metabolic process

GO:0005980 Glycogen catabolic process

GO:0051343 Positive regulation of cyclic-nucleotide phosphodiesterase activity

GO:0001975 Response to amphetamine

GO:1901841 Regulation of high voltage-gated calcium channel activity

GO:1901844 Regulation of cell communication by electrical coupling involved in

cardiac conduction

GO:0007190 Activation of adenylate cyclase activity

GO:0060316 Positive regulation of ryanodine-sensitive calcium-release channel activity

GO:0006936 Muscle contraction

GO:0043647 Inositol phosphate metabolic process

GO:0021762 Substantia nigra development

GO:0019233 Sensory perception of pain

GO:0060315 Negative regulation of ryanodine-sensitive calcium-release channel activity

GO:0032516 Positive regulation of phosphoprotein phosphatase activity

GO:0005513 Detection of $\mathrm{Ca}^{2+}$

GO:0010801 Negative regulation of peptidyl-threonine phosphorylation

GO:0051412 Response to corticosterone

GO:0010880 Regulation of release of sequestered $\mathrm{Ca}^{2+}$ into cytosol by sarcoplasmic reticulum

GO:0010881 Regulation of cardiac muscle contraction by regulation of the release of

sequestered $\mathrm{Ca}^{2+}$

GO:0060314 Regulation of ryanodine-sensitive calcium-release channel activity

GO:0055117 Regulation of cardiac muscle contraction

GO:0035307 Positive regulation of protein dephosphorylation

GO:0031954 Positive regulation of protein autophosphorylation

GO:0051000 Positive regulation of nitric-oxide synthase activity

GO:0032465 Regulation of cytokinesis

GO:0043547 Positive regulation of GTPase activity

GO:0001822 Kidney development

GO:0043065 Positive regulation of apoptotic process

GO:0050999 Regulation of nitric-oxide synthase activity

GO:0010800 Positive regulation of peptidyl-threonine phosphorylation

GO:0043388 Positive regulation of DNA binding

GO:0022400 Regulation of rhodopsin-mediated signaling pathway

GO:0002027 Regulation of heart rate

GO:0071902 Positive regulation of protein serine/threonine kinase activity

GO:0007223 Wnt signaling pathway, calcium-modulating pathway

GO:0045893 Positive regulation of transcription, DNA-templated

GO:0034704 Calcium channel complex

GO:0030017 Sarcomere

GO:0005876 Spindle microtubule

GO:0043274 Phospholipase binding

GO:0031997 N-terminal myristoylation domain binding

GO:0072542 Protein phosphatase activator activity

GO:0030235 Nitric-oxide synthase regulator activity

GO:0008440 Inositol-1,4,5-trisphosphate 3-kinase activity

GO:0031996 Thioesterase binding

GO:0031432 Titin binding

GO:0043539 Protein serine/threonine kinase activator activity

GO:0015276 Ligand-gated ion channel activity
$1.12 \times 10^{-4}$

$1.56 \times 10^{-4}$

$2.35 \times 10^{-4}$

$3.13 \times 10^{-4}$

$3.35 \times 10^{-4}$

$4.01 \times 10^{-4}$

$4.75 \times 10^{-4}$

$5.41 \times 10^{-4}$

$6.12 \times 10^{-4}$

$7.28 \times 10^{-4}$

$7.31 \times 10^{-4}$

$8.62 \times 10^{-4}$

$1.00 \times 10^{-3}$

$1.00 \times 10^{-3}$

$1.67 \times 10^{-3}$

$1.67 \times 10^{-3}$

$1.87 \times 10^{-3}$

$1.87 \times 10^{-3}$

$2.28 \times 10^{-3}$

$2.28 \times 10^{-3}$

$2.28 \times 10^{-3}$

$2.50 \times 10^{-3}$

$2.50 \times 10^{-3}$

$3.00 \times 10^{-3}$

$3.11 \times 10^{-3}$

$3.45 \times 10^{-3}$

$3.49 \times 10^{-3}$

$3.76 \times 10^{-3}$

$4.04 \times 10^{-3}$

$4.33 \times 10^{-3}$

$5.59 \times 10^{-3}$

$6.27 \times 10^{-3}$

$7.74 \times 10^{-3}$

$7.81 \times 10^{-3}$

$2.75 \times 10^{-3}$

$6.61 \times 10^{-3}$

$8.36 \times 10^{-3}$

$2.72 \times 10^{-5}$

$3.24 \times 10^{-5}$

$1.61 \times 10^{-4}$

$2.99 \times 10^{-4}$

$4.78 \times 10^{-4}$

$9.59 \times 10^{-4}$

$9.59 \times 10^{-4}$

$1.60 \times 10^{-3}$

$6.33 \times 10^{-3}$ 
Table I. Continued.

\begin{tabular}{|c|c|c|}
\hline Term and description & Count in gene set & P-value \\
\hline GO:0001105 RNA polymerase II transcription coactivator activity & 3 & $6.68 \times 10^{-3}$ \\
\hline GO:0019901 Protein kinase binding & 6 & $8.04 \times 10^{-3}$ \\
\hline hsa05010:Alzheimer's disease & 6 & $9.66 \times 10^{-4}$ \\
\hline hsa04915:Estrogen signaling pathway & 5 & $1.07 \times 10^{-3}$ \\
\hline hsa04922:Glucagon signaling pathway & 5 & $1.07 \times 10^{-3}$ \\
\hline hsa04916:Melanogenesis & 5 & $1.11 \times 10^{-3}$ \\
\hline hsa04020:Calcium signaling pathway & 6 & $1.28 \times 10^{-3}$ \\
\hline hsa04728:Dopaminergic synapse & 5 & $2.75 \times 10^{-3}$ \\
\hline hsa05214:Glioma & 4 & $3.29 \times 10^{-3}$ \\
\hline hsa05031:Amphetamine addiction & 4 & $3.43 \times 10^{-3}$ \\
\hline hsa04720:Long-term potentiation & 4 & $3.43 \times 10^{-3}$ \\
\hline hsa04744:Phototransduction & 3 & $6.84 \times 10^{-3}$ \\
\hline
\end{tabular}

B, Upregulated DEGs

Term and description

Count in gene set $\mathrm{P}$-value

GO:0007155 Cell adhesion

GO:0007010 Cytoskeleton organization

GO:0001501 Skeletal system development

GO:0045944 Positive regulation of transcription from RNA polymerase II promoter

GO:0008584 Male gonad development

GO:0002576 Platelet degranulation

GO:0042475 Odontogenesis of dentin-containing teeth

GO:0090190 Positive regulation of branching involved in ureteric bud morphogenesis

GO:0050679 Positive regulation of epithelial cell proliferation

\begin{tabular}{|c|c|}
\hline 13 & $1.02 \times 10^{-4}$ \\
\hline 7 & $1.01 \times 10^{-3}$ \\
\hline 6 & $2.92 \times 10^{-3}$ \\
\hline 16 & $3.94 \times 10^{-3}$ \\
\hline 5 & $4.48 \times 10^{-3}$ \\
\hline 5 & $6.18 \times 10^{-3}$ \\
\hline 4 & $6.96 \times 10^{-3}$ \\
\hline 3 & $7.87 \times 10^{-3}$ \\
\hline 4 & $8.85 \times 10^{-3}$ \\
\hline 29 & $8.21 \times 10^{-4}$ \\
\hline 45 & $8.97 \times 10^{-4}$ \\
\hline 10 & $1.05 \times 10^{-3}$ \\
\hline 8 & $4.34 \times 10^{-3}$ \\
\hline 9 & $5.57 \times 10^{-3}$ \\
\hline 4 & $6.37 \times 10^{-3}$ \\
\hline 21 & $6.61 \times 10^{-3}$ \\
\hline 8 & $6.92 \times 10^{-3}$ \\
\hline 3 & $7.39 \times 10^{-3}$ \\
\hline 19 & $7.89 \times 10^{-3}$ \\
\hline 11 & $2.55 \times 10^{-5}$ \\
\hline 5 & $7.42 \times 10^{-3}$ \\
\hline 4 & $9.33 \times 10^{-3}$ \\
\hline 9 & $9.83 \times 10^{-4}$ \\
\hline 6 & $1.72 \times 10^{-3}$ \\
\hline 11 & $2.13 \times 10^{-3}$ \\
\hline 8 & $3.71 \times 10^{-3}$ \\
\hline 11 & $5.44 \times 10^{-3}$ \\
\hline 9 & $7.47 \times 10^{-3}$ \\
\hline
\end{tabular}

GO:0016020 Membrane

GO:0005886 Plasma membrane

GO:0005856 Cytoskeleton

GO:0031012 Extracellular matrix

GO:0005925 Focal adhesion

GO:0031093 Platelet $\alpha$ granule lumen

GO:0005576 Extracellular region

GO:0005913 Cell-cell adhesion junction

GO:0043034 Costamere

GO:0005887 Integral component of plasma membrane

GO:0003779 Actin binding

GO:0005200 Structural constituent of cytoskeleton

GO:0050839 Cell adhesion molecule binding

hsa04510:Focal adhesion

hsa04512:ECM-receptor interaction

hsa04151:PI3K-Akt signaling pathway

hsa05205:Proteoglycans in cancer

hsa05200:Pathways in cancer

hsa05206:MicroRNAs in cancer

$7.47 \times 10^{-3}$

GO, Gene Ontology; KEGG, Kyoto Encyclopedia of Genes and Genomes; DEGs, differentially expressed genes; MM, malignant melanoma.

phase. Subsequently, mutant cells invade the dermal layer and trigger angiogenesis. This 'angiogenic switch' is involved in invasiveness and is characterized by the regulation of genes, including VEGFA, VEGF receptor genes and related angiogenic signaling pathways (27). However, the molecular pathways underlying melanoma remain largely unknown. 
Table II. Functional roles of the 10 hub genes with a degree $\geq 10$.

\begin{tabular}{|c|c|c|c|}
\hline No. & Gene symbol & Full name & Function \\
\hline 1 & $V E G F A$ & Vascular endothelial growth factor A & $\begin{array}{l}\text { Induction of proliferation and migration of vascular } \\
\text { endothelial cells; essential for both physiological as } \\
\text { well as pathological angiogenesis. }\end{array}$ \\
\hline 2 & $B A X$ & BCL2-associated X, apoptosis regulator & $\begin{array}{l}\text { It acts as either an anti- or proapoptotic regulator and is } \\
\text { involved in several cellular activities. }\end{array}$ \\
\hline 3 & CALMI & Calmodulin 1 & $\begin{array}{l}\text { Expression of a member of the EF-hand calcium-binding } \\
\text { protein family }\end{array}$ \\
\hline 4 & CALM2 & Calmodulin 2 & $\begin{array}{l}\text { Role in proliferation, cell cycle progression, and } \\
\text { signaling cascades. }\end{array}$ \\
\hline 5 & CALM3 & Calmodulin 3 & $\begin{array}{l}\text { Calcium binding; enzymatic co-factor; role in cell cycle } \\
\text { regulation as well as cytokinesis. }\end{array}$ \\
\hline 6 & FN1 & Fibronectin 1 & $\begin{array}{l}\text { Fibronectin expression; involvement in cell adhesion } \\
\text { and migration processes. }\end{array}$ \\
\hline 7 & PRKCA & Protein kinase $\mathrm{C} \alpha$ & $\begin{array}{l}\text { Essential roles in numerous cellular processes, including } \\
\text { cell volume control, checkpoints of the cell cycle, cell } \\
\text { adhesion, as well as cell transformation. }\end{array}$ \\
\hline 8 & $I G F 1$ & Insulin-like growth factor 1 & $\begin{array}{l}\text { Expression of protein with similar structure as well } \\
\text { as function to insulin; member of a family of proteins } \\
\text { involved in regulating growth and development. }\end{array}$ \\
\hline 9 & $R B 1$ & $\mathrm{RB}$ transcriptional corepressor 1 & $\begin{array}{l}\text { Expression of negative regulatory protein of the cell } \\
\text { cycle; the first tumor suppressor gene to be identified. }\end{array}$ \\
\hline 10 & $D E S$ & Desmin & $\begin{array}{l}\text { Expression of a muscle-specific class III intermediate } \\
\text { filament. Homopolymers of this protein form a stable } \\
\text { intracytoplasmic filamentous network which connects } \\
\text { myofibrils to each other as well as to the plasma } \\
\text { membrane. }\end{array}$ \\
\hline
\end{tabular}

The development of biomarkers with improved accuracy is essential for the effective diagnosis and treatment of melanoma. Genetic changes in melanoma may be observed using microarray technology, which may also be beneficial for the identification of novel biomarkers in other diseases.

The present study used $3 \mathrm{mRNA}$ microarray datasets to identify DEGs between malignant melanoma tissues and normal nevi tissues. A total of 182 DEGs were identified, which included 52 downregulated and 130 upregulated genes. DEG interactions were studied using GO and KEGG enrichment analyses. The upregulated genes were found to be associated with the cell membrane, cytoskeleton, extracellular region, actin binding, mitotic cell cycle and PI3K-Akt signaling cascades, while the downregulated genes were found to be involved in progressive regulation of transcription, protein kinase binding, melanogenesis, and the estrogen and calcium signaling pathways. Literature retrieval results indicated that the associations between malignant melanoma and these molecular mechanisms (oocyte meiosis, protein kinase binding and estrogen signaling pathway) have not been reported widely. A total of 10 DEGs with degrees $\geq 10$ in the PPI network were selected as hub genes. The PPI network revealed that $B A X$ directly interacts with $M Y C N, I G F 1$, $R U N X 1$ (runt-related transcription factor 1), MSH6, PRKDC (protein kinase, DNA-activated, catalytic subunit), $C A L M 1$,
CALM2, CALM3, TP63, VEGFA and $R B 1$, indicating a key role for $B A X$ in melanoma. Furthermore, the PPI network in DEGs is a novel gene interaction observed in malignant melanoma. It is well known that $V E G F A$ and $B A X$ are involved in tumor malignancy $(28,29)$, a finding confirmed in the present study. VEGFA is related to angiogenesis, a process which is required for tumor growth and metastasis (30). Vasculogenic mimicry (VM) is an endothelial vessel supply system in cancers that VM reflects the aggressive ability of tumor cells (31). Recently, the $c-M y c$ gene was reported to promote tumorigenesis of melanoma by promoting vasculogenic mimicry via the Bax signaling pathway (24). VEGFA overexpression has also been observed in lung, pancreatic and other cancers (32-34). In addition to its role in cell cycle progression, $V E G F A$ is a potent angiogenic factor, which is required for oncogenesis (35,36). CALM1, CALM2 and CALM3 belong to the CaM gene family $(37,38)$. They all encode a similar CaM protein, with differences at the nucleotide level. CaM serves an essential role in disease pathogenesis via the $\mathrm{Ca}^{2+}$ signaling pathway $(39,40)$. Furthermore, it is involved in apoptosis by balancing the expression levels of the proapoptotic protein, BAX, with those of the antiapoptotic protein, Bcl-2 (41).

Among the identified hub genes, $B A X$ overexpression was found to be associated with the lowest survival rate. The protein encoded by this gene is a part of the Bcl-2 protein family that 


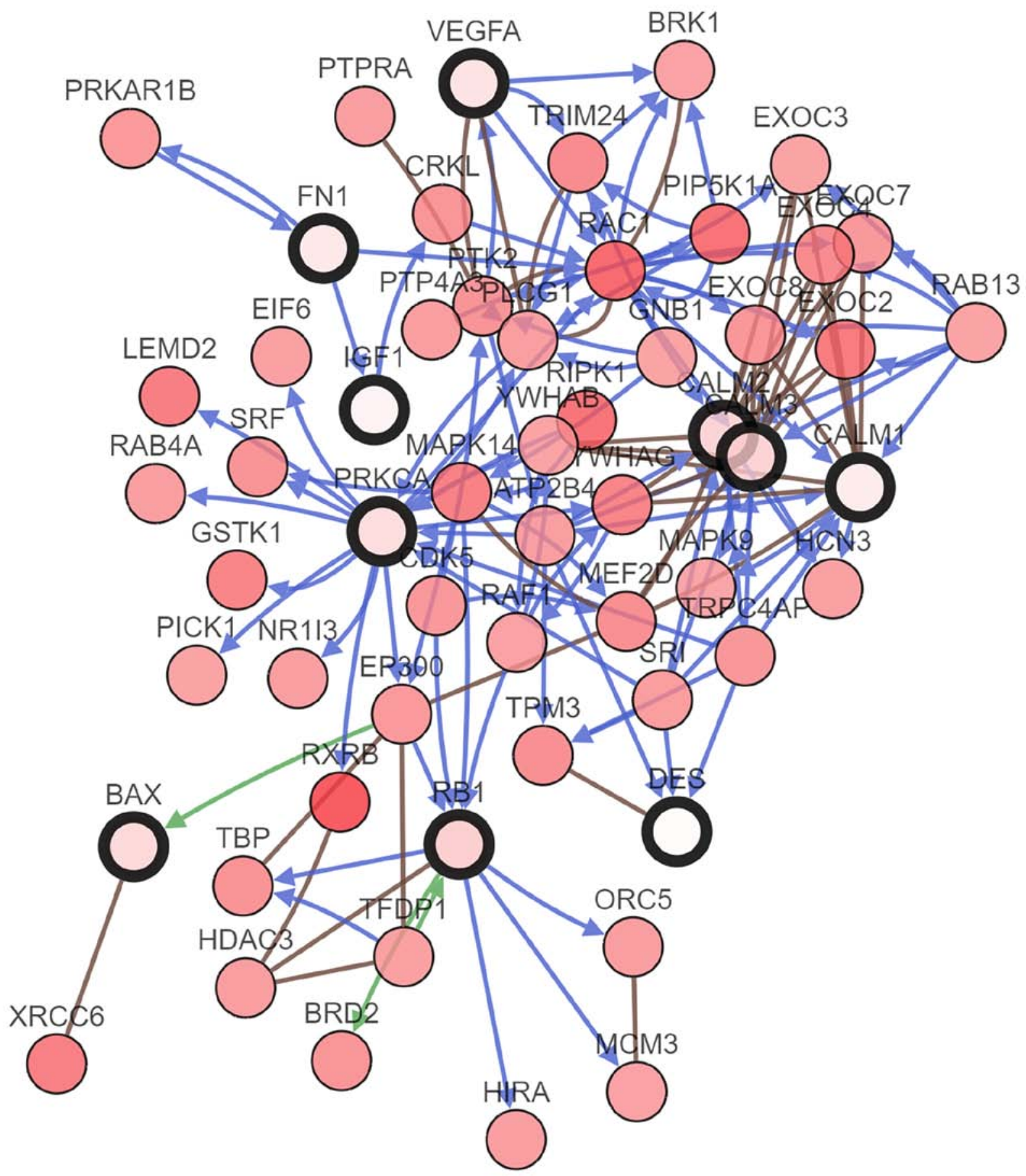

Figure 2. Hub gene interaction network. cBioPortal was used to examine the hub genes and their co-expression genes. Blue arrows represent controlling state change of genes, green arrows represent controlling expression of genes and brown lines represent complex with other genes. Nodes with a bold black outline represent the hub genes, while nodes with a thin black outline represent co-expression genes. DES, desmin; VEGFA, vascular endothelial growth factor A; $C A L M 1$, calmodulin 1; CALM2, calmodulin 2; CALM3, calmodulin 3; FN1, fibronectin 1; PRKCA, protein kinase C $\alpha$; IGF1, insulin-like growth factor 1; RB1, retinoblastoma transcriptional corepressor 1.

consists of antiapoptotic and proapoptotic members. $B A X$ gene expression is associated with shorter patient survival, chemoresistance and recurrence in melanoma $(42,43)$. Thus, it is regarded as a target for anticancer agents. The expression of the hub genes in relation to both overall and disease-free survival was evaluated. $B A X$ alteration was found to significantly affect both overall survival and disease-free survival. BAX could induce the decrease in overall survival and disease-free survival. Moreover, clinical studies have reported that a shorter survival period is significantly associated with $B A X$ gene overexpression $(44,45)$. However, the expression levels of the other hub genes in overall survival were not statistically significant compared to $B A X$. This result may have occurred due to the fact that survival analysis in cBioPortal is performed based on a relationship between gene mutation and prognosis. However, gene overexpression may arise via either mutation or amplification. Accordingly, hub gene overexpression in melanoma may occur due to gene amplification rather 


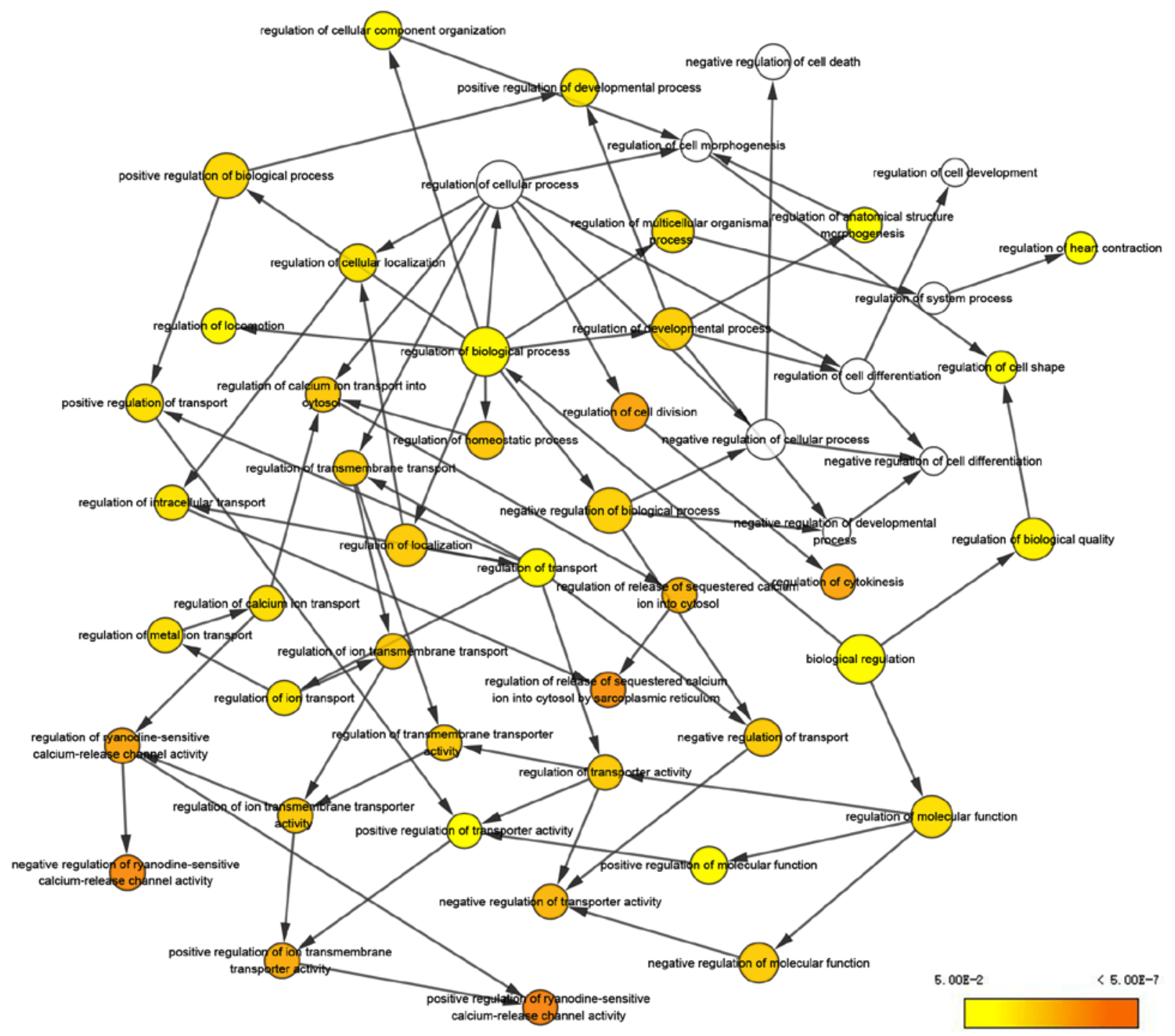

Figure 3. BiNGO analysis of the biological process of genes with a degree $\geq 10$. BiNGO was used for hub gene biological process analysis. The color depth of the nodes represents the corrected P-value of the ontologies. The magnitude of the nodes represents the genes that participate in the ontologies. BiNGO, Biological Networks Gene Oncology.

than mutation, thus creating the need for further research in order to confirm the association between melanoma and the hub genes. Oncomine analysis demonstrated that mRNA expression levels of $B A X, C A L M 1, C A L M 3, F N 1, P R K C A, R B 1$ and $V E G F A$ were higher in melanoma tissue than in normal nevi tissues, whereas the mRNA expression level of $I G F I$ was lower in melanoma tissues. Previous studies have reported that CALM2 levels in gastric, breast and other cancer tissues are higher than those in normal tissues, and that this gene may subsequently be used as a prognostic target (46-48). Oncomine analysis in melanoma indicated that CALM2 expression in melanoma was not higher. Further investigation is therefore required to confirm $C A L M 2$ expression in cancers. The DES gene encodes desmin, one of the first muscle-specific proteins to be expressed during the early phases of skeletal and cardiac muscle differentiation (49). Certain studies have suggested that desmin expression is elevated in colorectal cancer, and that it may be used as a novel prognostic predictor $(50,51)$. DES expression is increased in osteogenic melanoma, however the expression of $D E S$ is low in other types of melanoma (52).
Thus, the reason why there was no significant difference in the Oncomine database analysis results may be that the Oncomine database did not include the classification of melanoma. Similarly, FNI, PRKCA, RBI and IGFI had been reported to influence tumorigenesis and initiation in other types of cancer $(53,54)$, which was consistent with our study in melanoma. It was speculated that the $\mathrm{Ca}^{2+}$ signaling pathway is associated with malignant melanoma. The disruption of the homeostasis of this pathway during tumorigenesis leads to abnormal expression of $B A X(55)$. Disruption of $\mathrm{Ca}^{2+}$ signaling pathway homeostasis also promotes tumor angiogenesis via the overexpression of VEGFA.

There were certain limitations in the present study. Firstly, the GES3189 dataset comprised 45 melanoma samples and 18 normal nevi samples, whereas the sample quantity of the other two databases was insufficient. However, since three databases were used to choose the overlap in Venn diagram as DEGs, this may improve the credibility of the analysis results. Secondly, certain biomarkers associated with melanoma were identified; however, further experimental studies, including 
A
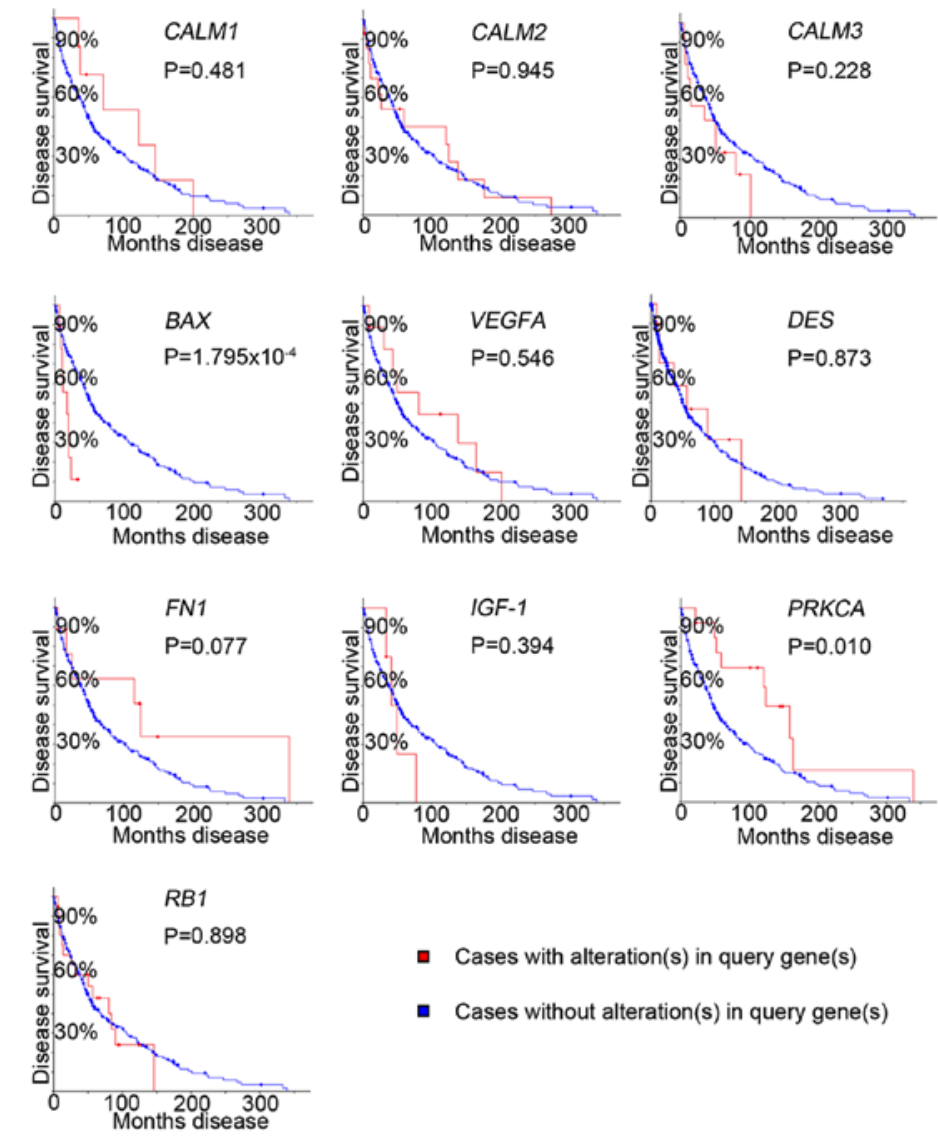

- Cases with alteration(s) in query gene(s)

- Cases without alteration(s) in query gene(s)

B
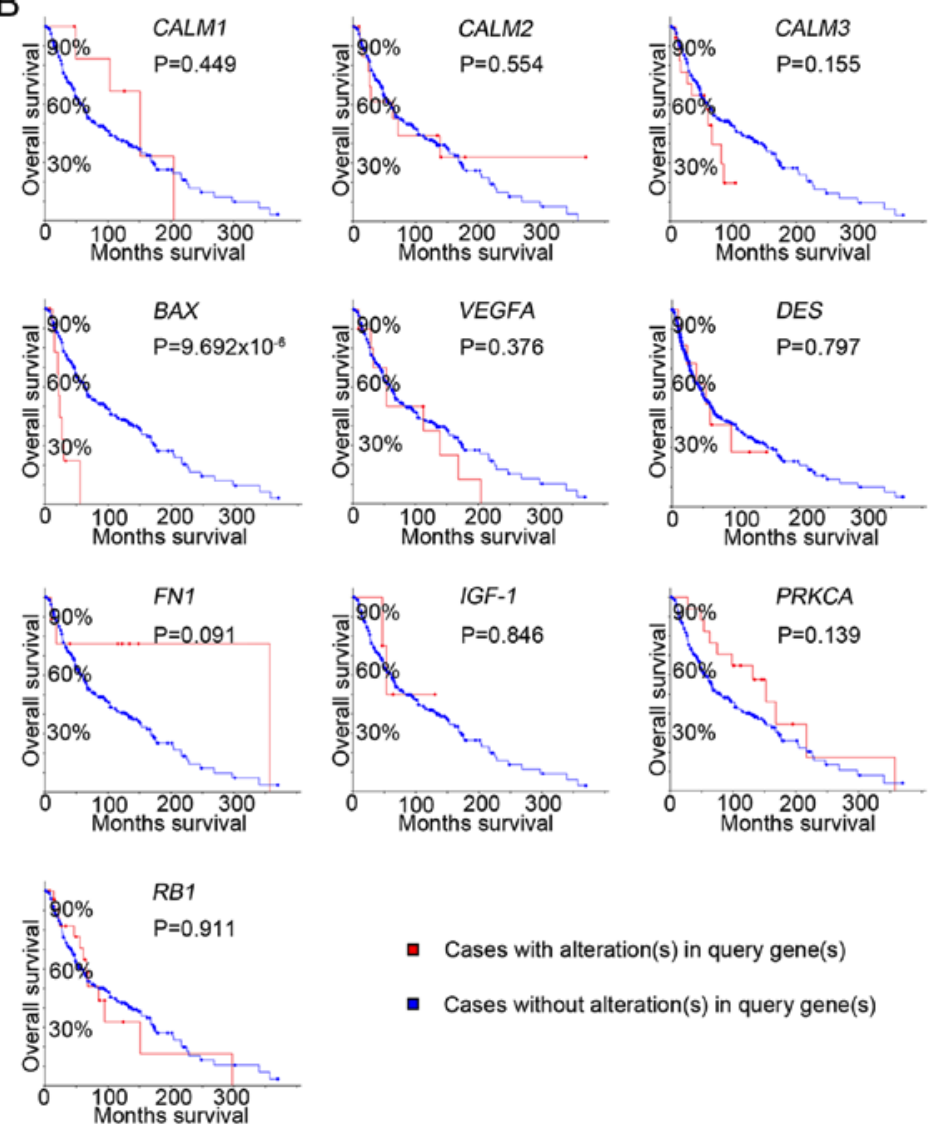

- Cases with alteration(s) in query gene(s)

- Cases without alteration(s) in query gene(s)

Figure 4. Disease-free survival and overall survival analyses of the hub genes using Kaplan-Meier analysis on the cBioPortal online platform. (A) Disease-free survival and (B) overall survival. $\mathrm{P}<0.05$ was considered to indicate a statistically significant difference. $D E S$, desmin; VEGFA, vascular endothelial growth factor A; CALM1, calmodulin 1; CALM2, calmodulin 2; CALM3, calmodulin 3; FN1, fibronectin 1; PRKCA, protein kinase C $\alpha$; IGF1, insulin-like growth factor $1 ; R B 1$, retinoblastoma transcriptional corepressor 1 . 

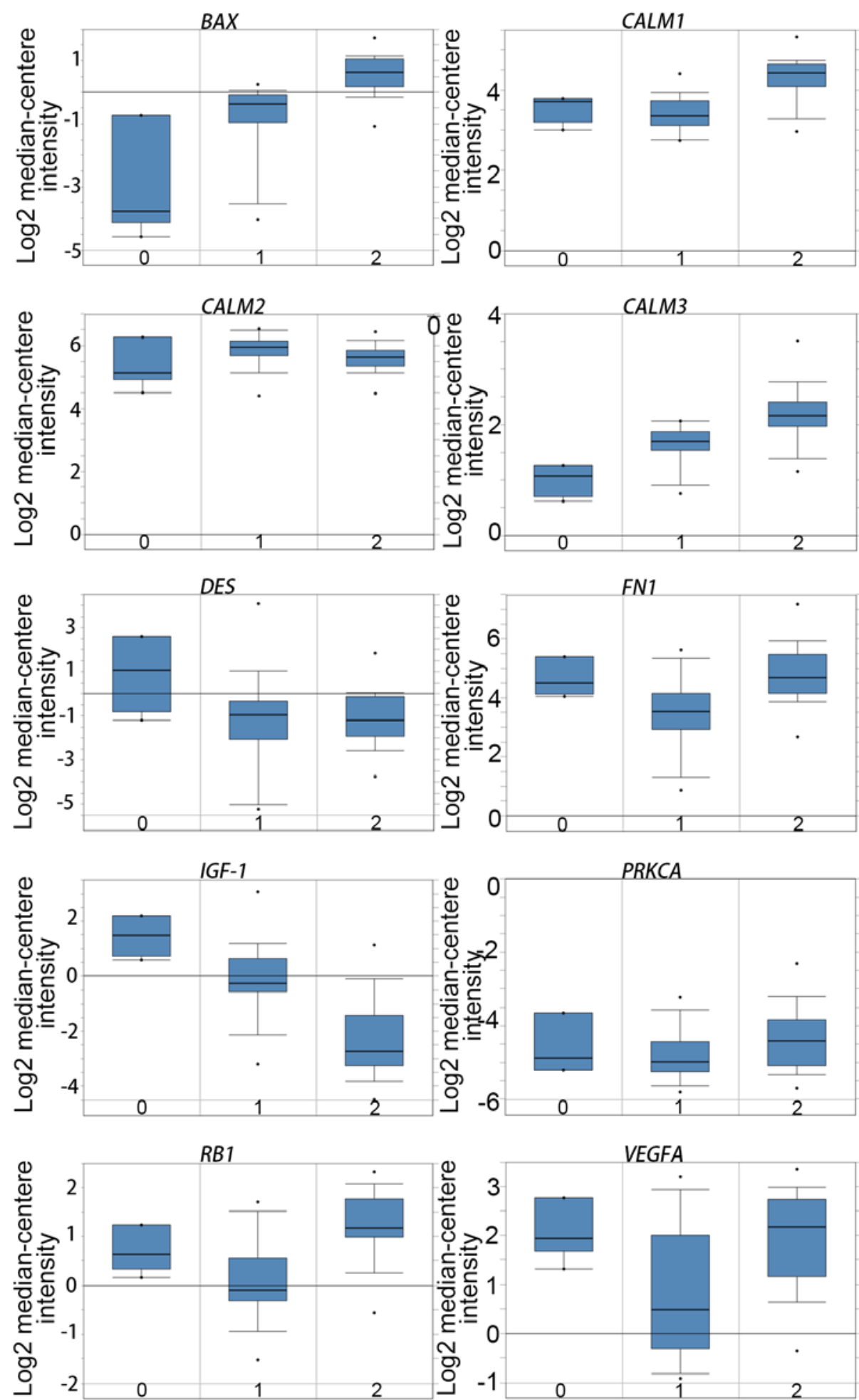

Figure 5. Expression of the hub genes in the GSE3189 dataset obtained using Oncomine online analysis. 0, No value (normal skin); 1, Skin nevus; 2, Cutaneous melanoma. DES, desmin; VEGFA, vascular endothelial growth factor A; CALM1, calmodulin 1; CALM2, calmodulin 2; CALM3, calmodulin 3; FN1, fibronectin $1 ; P R K C A$, protein kinase $\mathrm{C} \alpha ; I G F 1$, insulin-like growth factor $1 ; R B 1$, retinoblastoma transcriptional corepressor 1.

immunohistochemistry, animal testing and clinical trials, are required to validate these findings. Despite these limitations, there is few report that upregulation of the $C A L M$ gene family (CALM1, CALM2 and CALM3) in malignant melanoma was associated with poor prognosis. In addition, the present study was the first to report associations between the identified DEGs and hub gene interactions in malignant melanoma.
In conclusion, the present study identified DEGs that may be associated with the initiation or progression of melanoma. The 182 DEGs and 10 hub genes that were identified may be considered as potential biomarkers of melanoma. Nonetheless, additional research is required to further understand the biological functions of these genes in melanoma. 


\section{Acknowledgements}

The authors would like to thank Dr Xiangkun Wang from the Department of Hepatobiliary of The First Affiliated Hospital of Guangxi Medical University and Professor Jingming Zhao from Guangxi Key Laboratory of Regenerative Medicine for their kind suggestions for data analysis.

\section{Funding}

The present study was supported by National Natural Science Foundation of China (grant no. 81701938).

\section{Availability of data and materials}

The datasets generated and/or analyzed during the current study are available in the Gene Expression Omnibus repository, [GSE3189 (https://www.ncbi.nlm.nih.gov/geo/query/acc. cgi?acc=GSE3189),GSE4570(https://www.ncbi.nlm.nih.gov/geo/ query/acc.cgi?acc=GSE4570) and GSE4587 (https://www. ncbi.nlm.nih.gov/geo/query/acc.cgi?acc=GSE4587)].

\section{Authors' contributions}

GL conceived the study and wrote the manuscript. BL helped to design the study. GY conducted this study and interpreted the data. YY was responsible for data analysis. All authors approved the final manuscript to be published and agreed to be accountable for all aspects of the work in ensuring that questions related to the accuracy or integrity of any part of the work are appropriately investigated and resolved.

\section{Ethics approval and consent to participate}

Not applicable.

\section{Patient consent for publication}

Not applicable.

\section{Competing interests}

The authors declare that they have no competing interests.

\section{References}

1. Qin J, Li S, Zhang C, Gao DW, Li Q, Zhang H, Jin XD and Liu Y Apoptosis and injuries of heavy ion beam and $\mathrm{X}$-ray radiation on malignant melanoma cell. Exp Biol Med (Maywood) 242: 953-960, 2017.

2. Ci C, Tang B, Lyu D, Liu W, Qiang D, Ji X, Qiu X, Chen L and Ding W: Overexpression of CDCA8 promotes the malignant progression of cutaneous melanoma and leads to poor prognosis. Int J Mol Med 43: 404-412, 2019.

3. Bai X, Kong Y, Chi Z, Sheng X, Cui C, Wang X, Mao L, Tang B, Li S, Lian B, et al: MAPK pathway and TERT promoter gene mutation pattern and its prognostic value in melanoma patients: A retrospective study of 2,793 cases. Clin Cancer Res 23: 6120-6127, 2017.

4. Shalem O, Sanjana NE, Hartenian E, Shi X, Scott DA, Mikkelson T, Heckl D, Ebert BL, Root DE, Doench JG and Zhang F: Genome-scale CRISPR-Cas9 knockout screening in human cells. Science 343: 84-87, 2014.
5. Nissan MH, Pratilas CA, Jones AM, Ramirez R, Won H, Liu C, Tiwari S, Kong L, Hanrahan AJ, Yao Z, et al: Loss of NF1 in cutaneous melanoma is associated with RAS activation and MEK dependence. Cancer Res 74: 2340-2350, 2014.

6. Burd CE, Liu W, Huynh MV, Waqas MA, Gillahan JE Clark KS, Fu K, Martin BL, Jeck WR, Souroullas GP, et al: Mutation-specific RAS oncogenicity explains NRAS codon 61 selection in melanoma. Cancer Discov 4: 1418-1429, 2014.

7. Agrawal P, Fontanals-Cirera B, Sokolova E, Jacob S, Vaiana CA, Argibay D, Davalos V, McDermott M, Nayak S, Darvishian F, et al: A systems biology approach identifies FUT8 as a driver of melanoma metastasis. Cancer Cell 31: 804-819.e7, 2017.

8. Edgar R, Domrachev M and Lash AE: Gene expression omnibus: NCBI gene expression and hybridization array data repository. Nucleic Acids Res 30: 207-210, 2002.

9. Talantov D, Mazumder A, Yu JX, Briggs T, Jiang Y, Backus J, Atkins D and Wang Y: Novel genes associated with malignant melanoma but not benign melanocytic lesions. Clin Cancer Res 11: 7234-7242, 2005.

10. Hoek K, Rimm DL, Williams KR, Zhao H, Ariyan S, Lin A, Kluger HM, Berger AJ, Cheng E, Trombetta ES, et al: Expression profiling reveals novel pathways in the transformation of melanocytes to melanomas. Cancer Res 64: 5270-5282, 2004.

11. Smith AP, Hoek K and Becker D: Whole-genome expression profiling of the melanoma progression pathway reveals marked molecular differences between nevi/melanoma in situ and advanced-stage melanomas. Cancer Biol Ther 4: 1018-1029, 2005.

12. Benjamini $\mathrm{Y}$ and Hochberg Y: Controlling the false discovery rate: A practical and powerful approach to multiple testing. J R Stat Soc Ser B (Methodological) 57: 289-300, 1995.

13. Huang DW, Sherman BT, Tan Q, Collins JR, Alvord WG, Roayaei J, Stephens R, Baseler MW, Lane HC and Lempicki RA: The DAVID gene functional classification tool: A novel biological module-centric algorithm to functionally analyze large gene lists. Genome Biol 8: R183, 2007.

14. Kanehisa M, Furumichi M, Tanabe M, Sato Y and Morishima K: KEGG: New perspectives on genomes, pathways, diseases and drugs. Nucleic Acids Res 45: D353-D361, 2017.

15. Ashburner M, Ball CA, Blake JA, Botstein D, Butler $H$, Cherry JM, Davis AP, Dolinski K, Dwight SS, Eppig JT, et al: Gene ontology: Tool for the unification of biology. The gene ontology consortium. Nat Genet 25: 25-29, 2000.

16. Szklarczyk D, Morris JH, Cook H, Kuhn M, Wyder S, Simonovic M, Santos A, Doncheva NT, Roth A, Bork P, et al: The STRING database in 2017: Quality-controlled protein-protein association networks, made broadly accessible. Nucleic Acids Res 45: D362-D368, 2017.

17. Shannon P, Markiel A, Ozier O, Baliga NS, Wang JT, Ramage D, Amin N, Schwikowski B and Ideker T: Cytoscape: A software environment for integrated models of biomolecular interaction networks. Genome Res 13: 2498-2504, 2003.

18. Bandettini WP, Kellman P, Mancini C, Booker OJ, Vasu S, Leung SW, Wilson JR, Shanbhag SM, Chen MY and Arai AE: MultiContrast delayed enhancement (MCODE) improves detection of subendocardial myocardial infarction by late gadolinium enhancement cardiovascular magnetic resonance: A clinical validation study. J Cardiovasc Magn Reson 14: 83, 2012.

19. Cerami E, Gao J, Dogrusoz U, Gross BE, Sumer SO, Aksoy BA, Jacobsen A, Byrne CJ, Heuer ML, Larsson E, et al: The cBio cancer genomics portal: An open platform for exploring multidimensional cancer genomics data. Cancer Discov 2: 401-404, 2012.

20. Gao J, Aksoy BA, Dogrusoz U, Dresdner G, Gross B, Sumer SO, Sun Y, Jacobsen A, Sinha R, Larsson E, et al: Integrative analysis of complex cancer genomics and clinical profiles using the cBioPortal. Sci Signal 6: pl1, 2013.

21. Maere S, Heymans K and Kuiper M: BiNGO: A Cytoscape plugin to assess overrepresentation of gene ontology categories in biological networks. Bioinformatics 21: 3448-3449, 2005.

22. Rhodes DR, Kalyana-Sundaram S, Mahavisno V, Varambally R, Yu J, Briggs BB, Barrette TR, Anstet MJ, Kincead-Beal C, Kulkarni P, et al: Oncomine 3.0: Genes, pathways, and networks in a collection of 18,000 cancer gene expression profiles. Neoplasia 9: 166-180, 2007.

23. Rhodes DR, Yu J, Shanker K, Deshpande N, Varambally R, Ghosh D, Barrette T, Pandey A and Chinnaiyan AM: ONCOMINE: A cancer microarray database and integrated data-mining platform. Neoplasia 6: 1-6, 2004. 
24. Lin X, Sun R, Zhao X, Zhu D, Zhao X, Gu Q, Dong X, Zhang D, Zhang Y, Li Y and Sun B: C-myc overexpression drives melanoma metastasis by promoting vasculogenic mimicry via c-myc/snail/Bax signaling. J Mol Med (Berl) 95: 53-67, 2017.

25. Brecht IB, Garbe C, Gefeller O, Pfahlberg A, Bauer J Eigentler TK, Offenmueller S, Schneider DT and Leiter U: 443 paediatric cases of malignant melanoma registered with the German central malignant melanoma registry between 1983 and 2011. Eur J Cancer 51: 861-868, 2015.

26. Shimizu A, Kaira K, Yasuda M, Asao $\mathrm{T}$ and Ishikawa $\mathrm{O}$ Decreased expression of class III $\beta$-tubulin is associated with unfavourable prognosis in patients with malignant melanoma. Melanoma Res 26: 29-34, 2016.

27. Song S, Jacobson KN, McDermott KM, Reddy SP, Cress AE, Tang H, Dudek SM, Black SM, Garcia JG, Makino A and Yuan JX: ATP promotes cell survival via regulation of cytosolic $[\mathrm{Ca} 2+]$ and Bcl-2/Bax ratio in lung cancer cells. Am J Physiol Cell Physiol 310: C99-S114, 2016.

28. Shi L, Zhang G, Zheng Z, Lu B and Ji L: Andrographolide reduced VEGFA expression in hepatoma cancer cells by inactivating HIF-1 $\alpha$ : The involvement of JNK and MTA1/HDCA Chem Biol Interact 273: 228-236, 2017.

29. Merino D, Lok SW, Visvader JE and Lindeman GJ: Targeting BCL-2 to enhance vulnerability to therapy in estrogen receptor-positive breast cancer. Oncogene 35: 1877-1887, 2016.

30. Zhong Z, Huang M, Lv M, He Y, Duan C, Zhang L and Chen J: Circular RNA MYLK as a competing endogenous RNA promotes bladder cancer progression through modulating VEGFA/VEGFR2 signaling pathway. Cancer Lett 403: 305-317, 2017.

31. Delgado-Bellido D, Serrano-Saenz S, Fernandez-Cortés M and Oliver FJ: Vasculogenic mimicry signaling revisited: Focus on non-vascular VE-cadherin. Mol Cancer 16: 65, 2017.

32. Lin CY, Cho CF, Bai ST, Liu JP, Kuo TT, Wang LJ, Lin YS, Lin CC, Lai LC, Lu TP, et al: ADAM9 promotes lung cancer progression through vascular remodeling by VEGFA, ANGPT2, and PLAT. Sci Rep 7: 15108, 2017.

33. Kim M, Jang K, Miller P, Picon-Ruiz M, Yeasky TM, El-Ashry D and Slingerland JM: VEGFA links self-renewal and metastasis by inducing Sox 2 to repress miR-452, driving Slug. Oncogene 36: 5199-5211, 2017.

34. Fahmy K, Gonzalez A, Arafa M, Peixoto P, Bellahcène A, Turtoi A, Delvenne P, Thiry M, Castronovo V and Peulen O: Myoferlin plays a key role in VEGFA secretion and impacts tumor-associated angiogenesis in human pancreas cancer. Int J Cancer 138: 652-663, 2016.

35. Cheng CY, Ho TY, Hsiang CY, Tang NY, Hsieh CL, Kao ST and Lee YC: Angelica sinensis Exerts Angiogenic and Anti-apoptotic effects against cerebral ischemia-reperfusion injury by activating p38MAPK/HIF-1[Formula: See text]/VEGF-A signaling in rats. Am J Chin Med 45: 1683-1708, 2017.

36. Zhang L, Lv Z, Xu J, Chen C, Ge Q, Li P, Wei D, Wu Z and Sun X: MicroRNA-134 inhibits osteosarcoma angiogenesis and proliferation by targeting the VEGFA/VEGFR1 pathway. FEBS J 285: 1359-1371, 2018

37. Boczek NJ, Gomez-Hurtado N, Ye D, Calvert ML, Tester DJ, Kryshtal D, Hwang HS, Johnson CN, Chazin WJ, Loporcaro CG, et al: Spectrum and prevalence of CALM1-, CALM2- and CALM3-encoded calmodulin variants in long QT syndrome and functional characterization of a novel long QT syndrome-associated calmodulin missense variant, E141G. Circ Cardiovasc Genet 9: 136-146, 2016.

38. Limpitikul WB, Dick IE, Tester DJ, Boczek NJ, Limphong P, Yang W, Choi MH, Babich J, DiSilvestre D, Kanter RJ, et al: A precision medicine approach to the rescue of function on malignant Calmodulinopathic long-QT syndrome. Circ Res 120: 39-48, 2017.

39. Cai R, Zhang C, Zhao Y, Zhu K, Wang Y, Jiang H, Xiang Y and Cheng B: Genome-wide analysis of the IQD gene family in maize. Mol Genet Genomics 291: 543-558, 2016.
40. Bürstenbinder K, Möller B, Plötner R, Stamm G, Hause G, Mitra D and Abel S: The IQD family of Calmodulin-binding proteins links calcium signaling to microtubules, membrane subdomains and the nucleus. Plant Physiol 173: 1692-1708, 2017.

41. Shoshan-Barmatz V, Krelin Y and Shteinfer-Kuzmine A VDAC1 functions in $\mathrm{Ca}^{2+}$ homeostasis and cell life and death in health and disease. Cell Calcium 69: 81-100, 2018.

42. Liu Z, Ding Y, Ye N, Wild C, Chen H and Zhou J: Direct activation of bax protein for cancer therapy. Med Res Rev 36: 313-341, 2016.

43. Gil J, Ramsey D, Szmida E, Leszczynski P, Pawlowski P, Bebenek M and Sasiadek MM: The BAX gene as a candidate for negative autophagy-related genes regulator on mRNA levels in colorectal cancer. Med Oncol 34: 16, 2017.

44. Del Principe MI, Dal Bo M, Bittolo T, Buccisano F, Rossi FM, Zucchetto A, Rossi D, Bomben R, Maurillo L, Cefalo M, et al: Clinical significance of bax/bcl-2 ratio in chronic lymphocytic leukemia. Haematologica 101: 77-85, 2016.

45. Kowalczyk AE, Krazinski BE, Godlewski J, Kiewisz J, Kwiatkowski P, Sliwinska-Jewsiewicka A, Kiezun J, Sulik M and Kmiec Z: Expression of the EP300, TP53 and BAX genes in colorectal cancer: Correlations with clinicopathological parameters and survival. Oncol Rep 38: 201-210, 2017.

46. Rust R, Visser L, van der Leij J, Harms G, Blokzijl T, Deloulme JC, van der Vlies P, Kamps W, Kok K, Lim M, et al: High expression of calcium-binding proteins, S100A10, S100A11 and CALM2 in anaplastic large cell lymphoma. Br J Haematol 131: 596-608, 2005.

47. Haddad SA, Lunetta KL, Ruiz-Narvaez EA, Bensen JT, Hong CC, Sucheston-Campbell LE, Yao S, Bandera EV, Rosenberg L, Haiman CA, et al: Hormone-related pathways and risk of breast cancer subtypes in African American women. Breast Cancer Res Treat 154: 145-154, 2015.

48. Cai H, Xu J, Han Y, Lu Z, Han T, Ding Y and Ma L: Integrated miRNA-risk gene-pathway pair network analysis provides prognostic biomarkers for gastric cancer. Onco Targets Ther 9: 2975-2986, 2016

49. Li CF, Yan ZK, Chen LB, Jin JP and Li DD: Desmin detection by facile prepared carbon quantum dots for early screening of colorectal cancer. Medicine (Baltimore) 96: e5521, 2017.

50. Wang Y, Li Y, Chen Z, Wang T, Gu J, Wu X, Yin Y, Wang M and Pan Z: The evaluation of colorectal cancer risk in serum by anti-DESMIN-conjugated CdTe/CdS quantum dots. Clin Lab 63: 579-586, 2017.

51. Ekinci O, Ogut B, Celik B and Dursun A: Compared with elastin Stains, h-Caldesmon and desmin offer superior detection of vessel invasion in gastric, pancreatic and colorectal adenocarcinomas. Int J Surg Pathol 26: 318-326, 2018.

52. Trevisan F, Tregnago AC, Lopes Pinto CA, Urvanegia ACM, Morbeck DL, Bertolli E, Riva Neto FR, Duprat Neto JP and de Macedo MP: Osteogenic melanoma with desmin expression. Am J Dermatopathol 39: 528-533, 2017.

53. Ku SY, Rosario S, Wang Y, Mu P, Seshadri M, Goodrich ZW, Goodrich MM, Labbé DP, Gomez EC, Wang J, et al: Rb1 and Trp53 cooperate to suppress prostate cancer lineage plasticity, metastasis and antiandrogen resistance. Science 355: 78-83, 2017.

54. Wang X, Zhu Q, Lin Y, Wu L, Wu X, Wang K, He Q, Xu C, Wan $X$ and Wang X: Crosstalk between TEMs and endothelial cells modulates angiogenesis and metastasis via IGF1-IGF1R signalling in epithelial ovarian cancer. Br J Cancer 117: 1371-1382, 2017

55. Ohshima Y, Takata N, Suzuki-Karasaki M, Yoshida Y, Tokuhashi Y and Suzuki-Karasaki Y: Disrupting mitochondrial $\mathrm{Ca} 2+$ homeostasis causes tumor-selective TRAIL sensitization through mitochondrial network abnormalities. Int J Oncol 51: 1146-1158, 2017.

This work is licensed under a Creative Commons Attribution-NonCommercial-NoDerivatives 4.0 International (CC BY-NC-ND 4.0) License. 